\title{
Assessing the Evolution of Physical Fitness in Children and Adolescents for Evidence-Based Teaching
}

\author{
Dario Colella, Domenico Monacis \\ Department of Humanities, Cultural Heritage, Education Sciences, University of Foggia, Foggia, Italy \\ Email: dario.colella@unifg.it,Domenico.monacis@unifg.it
}

How to cite this paper: Colella, D., \& Monacis, D. (2021). Assessing the Evolution of Physical Fitness in Children and Adolescents for Evidence-Based Teaching. Advances in Physical Education, 11, 183-194. https://doi.org/10.4236/ape.2021.112014

Received: January 28, 2021

Accepted: May 3, 2021

Published: May 6, 2021

Copyright $\odot 2021$ by author(s) and Scientific Research Publishing Inc. This work is licensed under the Creative Commons Attribution International License (CC BY 4.0).

http://creativecommons.org/licenses/by/4.0/

\section{(c) (i) Open Access}

\begin{abstract}
Physical fitness is one of the most important health indicators in children and adolescents. This study aims to assess the evolution of physical fitness levels in children and adolescents (9 - 14 yrs) related to weight-status, providing evidence-based instructions for physical education teachers, and promoting the quality of physical education programs. The sample consisted of 542 early adolescents aged 9 - 14 years $(M=269, F=273)$ recruited from secondary schools in Italy. Physical fitness was assessed with the following motor test: SBJ, MBT, $10 \times 5$ and One Mile RWt. In addition to descriptive statistics, independent samples T-test and factorial ANOVA were carried out to compare and assess the evolution of motor performances over time. Multiple linear regression analysis was executed to investigate the variance in motor test explained by weight and height. Results evidence a significant increase in SBJ and MBT, but not in $10 \times 5$ and OneMile Run/Walk test, in about all age groups, both in male and female, normal weight and overweight/obese. Data analysis suggests that strength test in which is required single movement execution is more associated and conditioned by individual growth and anthropometrics factors than test required repeated movement and greater coordinative engagement.
\end{abstract}

\section{Keywords}

Quality of Physical Education, Physical Fitness, Teaching Styles, Regional Observatory

\section{Introduction}

The increase of overweight and obesity in children and adolescents is one of the most important health-global priorities (Abarca-Gómez et al., 2017), having a 
significant effect on adult diabetes development (Zhang et al., 2019), increasing common chronic diseases in middle age (Yu et al., 2018) and specific causes of death in adult age (Bhaskaran et al., 2018).

Health benefits of physical activity and fitness levels are well documented and include not only the prevention of overweight and obesity (Al-Khudairy et al., 2017), cardiovascular (Abrignani et al., 2019) muscle and skeletal health improvement (Alves \& Alves, 2019), but are also associated with mental health and well-being (Hosker et al., 2019), cognitive and academic performances (Singh et al., 2019).

The new guidelines of World Health Organization suggest that children and adolescents should be active for at least 60 minutes daily of moderate to vigorous aerobic physical activity (MVPA) and regular strength activity (Bull et al., 2020). Unfortunately, about $85 \%$ of girls and $78 \%$ of boys aged $11-17$ do not meet international physical activity guidelines, and the percentage of obese children and adolescents has increased exponentially over the last 40 years: in 2016, over 50 million girls and 74 million boys (about the $6 \%-7 \%$ of teenagers) were overweight or obese (Guthold et al., 2020).

Studies reveal a progressive decline in physical fitness levels among children and adolescents especially in the transition from middle to high school (Pate et al., 2019); children with high overweight/obese BMI show lower performances than those with normal weight BMI, with more significant differences in boys rather than girls (Colley et al., 2019; Dong et al., 2019; Milanovic et al., 2019).

Motor competences and physical fitness levels acquired by children in primary schools are correlated with different developmental pathways: low motor competences are correlated with low physical fitness levels, both predictors of overweight and obesity in adulthood (Rodrigues et al., 2016; Utesch et al., 2019).

The triad of physical inactivity in children (Faigenbaum et al., 2018) identifies three distinct but related factors: exercise deficit disorders, pediatric dynapenia, motor illiteracy, generating a dangerous circular process: subjects with low levels of daily physical activity are less likely to participate in physical activities, even free/unstructured, which involve enjoyment and fun; this determines a lower predisposition to physical or sports practice and to a progressive reduction of the individual motor skills repertoire and the consequent levels of individual motor development.

School-based-intervention program and physical education represent an important area in which motor development, the evolution of motor abilities and motor skills learning integrate each other allowing the achievement of interdisciplinary and transversal objectives related to correct eating habits and healthy lifestyles (Yuksel et al., 2020).

Sedentary habits in developmental age, in particular in the last fifteen years, in addition to being one of the causes of the progressive increase in overweight and obesity in this age group, are among the main causes of the decline in levels of physical activity and motor performance coordinative and conditional, with worrying repercussions, both quantitative and qualitative, on organic, cogni- 
tive and social relations development, requiring specific methodological and well-oriented interventions (Myer et al., 2015).

In Italy, the percentage of obese children and adolescents is almost tripled from 1975 to 2016. As regards the percentage of obese children and adolescents, Italy ranks 61 st worldwide for females and 46th for males, occupying 6th and 8th place respectively in high-income countries (Guthold, et al., 2020).

The Regional Observatory on the monitoring of motor development of adolescents, at the University of Foggia, Didactics Laboratory of Motor Activities, involves numerous schools and arises from the need to periodically acquire, annually, quantitative and qualitative data on the development of health-related motor skills and physical activity levels in developmental age. These data constitute direct information on the evolution of children's motor skills in a geographical area and, indirectly, on the degree of effectiveness of the interventions carried out in schools and on the quality of physical education in secondary schools; promote the self-assessment of motor performance and health conditions by students, a prerequisite for education in daily motor activities.

The aim of this study is to assess the evolution of physical fitness levels in children and adolescents (9-14 yrs) related to weight-status, interpreting data to raise physical education teaching process.

\section{Materials and Methods}

\subsection{Sample}

The sample consists of 542 early adolescents aged 9 - 14 years $(M=269, F=273)$ recruited from secondary schools in Foggia, South of Italy. The following research project (2019-2021), approved by the Administration of the Puglia Region (southern Italy), concerns the implementation of the Regional Observatory on the monitoring of physical efficiency related to adolescent health, involves secondary schools and it will be coordinated by the University of Foggia (Italy) degree course in Sciences and Techniques of Preventive and Adapted Motor Activities-Laboratory of Didactics of Motor Activities. Children and parents were informed about the study purposes and contents and written informed consent was obtained from one parent. After detecting anthropometrics data (height, weight and BMI), participants were divided into different groups according to gender, BMI Cutoff (Normal-weight vs Overweight-Obese) and age group (9 10 yrs, $11-12$ yrs and $13-14$ yrs). Cole's scale (2000) was used to determine if children were classified as Normal Weight or Overweight/Obese. Table 1 shows main children's anthropometrics characteristics.

\subsection{Procedure and Assessment}

Children were evaluated during physical education lessons by PE teachers from January to March 2020. Process monitoring has been conducted with the supervision of the University of Foggia. The following motor tests were assessed: standing broad jump (SBJ) and medicine ball throw (MBT) to evaluate strength 
Table 1. Descriptive statistics and differences in mean of anthropometric characteristics.

\begin{tabular}{ccccccc}
\hline Gender & Group & Age & $\mathrm{N}$ & Height & Weight & BMI \\
\hline Male & $\mathrm{Nw}$ & $9-10$ & 39 & $1.45 \pm 0.07$ & $37.32 \pm 5.19$ & $17.47 \pm 1.74$ \\
& $\mathrm{Ow}-\mathrm{Ob}$ & $9-10$ & 44 & $1.49 \pm 0.09$ & $54.40 \pm 7.94$ & $21.34 \pm 1.56$ \\
& $\mathrm{Nw}$ & $11-12$ & 36 & $1.51 \pm 0.10$ & $41.71 \pm 8.32$ & $17.97 \pm 1.93$ \\
& $\mathrm{Ow}-\mathrm{Ob}$ & $11-12$ & 57 & $1.58 \pm 0.07$ & $61.41 \pm 9.43$ & $24.40 \pm 2.75$ \\
& $\mathrm{Nw}$ & $13-14$ & 59 & $1.63 \pm 0.08$ & $51.16 \pm 8.22$ & $18.97 \pm 1.91$ \\
& $\mathrm{Ow}-\mathrm{Ob}$ & $13-14$ & 34 & $1.63 \pm 0.08$ & $67.78 \pm 9.42$ & $25.35 \pm 2.81$ \\
& $\mathrm{Nw}$ & $9-10$ & 55 & $1.48 \pm 0.08$ & $38.89 \pm 6.54$ & $17.62 \pm 2.00$ \\
& $\mathrm{Ow}-\mathrm{Ob}$ & $9-10$ & 33 & $1.48 \pm 0.08$ & $53.81 \pm 9.71$ & $24.21 \pm 2.87$ \\
& $\mathrm{Nw}$ & $11-12$ & 54 & $1.54 \pm 0.08$ & $44.13 \pm 9.96$ & $18.34 \pm 2.99$ \\
& $\mathrm{Ow}-\mathrm{Ob}$ & $11-12$ & 33 & $1.55 \pm 0.07$ & $59.91 \pm 8.29$ & $24.75 \pm 2.91$ \\
& $\mathrm{Nw}$ & $13-14$ & 60 & $1.58 \pm 0.06$ & $47.82 \pm 6.01$ & $19.08 \pm 1.90$ \\
& $\mathrm{Ow}-\mathrm{Ob}$ & $13-14$ & 38 & $1.60 \pm 0.07$ & $66.81 \pm 8.29$ & $25.95 \pm 3.04$ \\
\hline
\end{tabular}

of the lower and upper limbs, shuttle run $10 \times 5(10 \times 5)$ and OneMile Run/Walk test (OneMile RWt) to evaluate resistance. For each test were performed two trials, reported the best results.

\subsection{Statistical Analysis}

After verified normal distribution of data with Saphiro-Wilk test, descriptive statistic was carried out presenting results as Means \pm SD. Independent samples T-test (Student's test) was performed to compare motor performances between $\mathrm{Nw}$ and $\mathrm{Ow}-\mathrm{Ob}$ groups, according to gender and age group. Cohen's $d$ value was carried out to measure the strength of the relationship between two variables (effect size): Very small (less than or equal to 0.20 ), Small (from 0.20 to 0.50 ), Medium (from 0.50 to 0.80 ) and Large (greater than 0.80), according to Cohen (1988). After verifying homoscedasticity condition (Levene's test), factorial ANOVA 2 (Nw vs $\mathrm{Ow}-\mathrm{Ob}) \times 3$ (9-10 vs $11-12$ vs $13-14) \times 2$ (Male vs Female) was carried out to examine the evolution in motor performances over time, and Fisher-test was used to analyze difference between means. Partial eta squared was also calculated, interpreting data with the following scale: Very small (less than or equal to 0.01), Small (from 0.01 to 0.06 ), Medium (from 0.06 to 0.14 ) and Large (greater than 0.14 ), according to Pierce et al. (2004). In addition, post-hoc test (Tuckey S-D) was executed to investigate which age's group shows higher significant differences. A stepwise multiple linear regression analysis was executed to investigate the variance in motor test explained by weight and height. In addition, partial $(r)$, and semi-partial correlation $(s r)$ were carried out to better explain variance between variables. All significance levels were set at $p<0.05$.

\section{Results}

Table 2 summarizes the results of motor test. Normal weight group shows better 
performances in all motor tests, except for MBT.

Table 3 reports statistical significative differences between $\mathrm{Nw}$ and $\mathrm{Ow}-\mathrm{Ob}$ groups. SBJ and One Mile RWt data show more statistical significance than MBT and $10 \times 5$. Large levels of effect size $(d>0.80)$ were reported in male $9-10 \mathrm{yrs}$, male 13 - 14 yrs and female 9 - 10 years for SBJ (respectively $d=1.27, d=0.98, d$ $=0.86)$; female 11 - 12 yrs showed large effect size in MBT $(d=0.83)$ and male 11 - 12 yrs in One Mile RwT $(d=0.93)$. Medium levels of effect size can be evidenced in male 11 - 12 yrs and female 13 - 14 yrs for SBJ, male 11 - 12 yrs for MBT, all $10 \times 5$ shuttle run test.

Factorial ANOVA between results is presented in Table 4. Data analysis shows how results are statistically different according to gender, BMI and age group independently, but not combined together. Particularly, gender influenced all motor test with medium to large value of partial eta squared $\left(\eta_{p}^{2}>\right.$ $0.14)$, BMI influenced SBJ $\left(\eta_{p}^{2}=0.126\right)$ and One Mile RWt $\left(\eta_{p}^{2}=0.072\right)$ and age group influenced SBJ and $\operatorname{MBT}\left(\eta_{p}^{2}=0.090, \eta_{p}^{2}=0.256\right)$.

Table 2. Descriptive statistics and differences in mean of the results of the SBJ, MBT, $10 \times$ 5 and one mile RWt.

\begin{tabular}{|c|c|c|c|c|c|c|}
\hline Gender & Group & Age & $\mathrm{SBJ}(\mathrm{m})$ & MBT (m) & $10 \times 5(\mathrm{sec})$ & One Mile RWt (m) \\
\hline \multirow[t]{6}{*}{ Male } & $\mathrm{Nw}$ & $9-10$ & $1.46 \pm 0.17$ & $3.89 \pm 0.75$ & $21.16 \pm 2.88$ & $9.87 \pm 1.73$ \\
\hline & $\mathrm{Ow}-\mathrm{Ob}$ & $9-10$ & $1.22 \pm 0.19$ & $4.17 \pm 1.00$ & $22.48 \pm 3.05$ & $11.21 \pm 2.61$ \\
\hline & $\mathrm{Nw}$ & $11-12$ & $1.50 \pm 0.23$ & $4.63 \pm 0.99$ & $20.65 \pm 2.52$ & $9.18 \pm 2.39$ \\
\hline & $\mathrm{Ow}-\mathrm{Ob}$ & $11-12$ & $1.35 \pm 0.26$ & $5.13 \pm 1.02$ & $2213 \pm 3.13$ & $11.14 \pm 1.81$ \\
\hline & $\mathrm{Nw}$ & $13-14$ & $1.71 \pm 0.27$ & $5.90 \pm 1.46$ & $20.08 \pm 2.82$ & $8.67 \pm 21.50$ \\
\hline & $\mathrm{Ow}-\mathrm{Ob}$ & $13-14$ & $1.46 \pm 0.19$ & $6.02 \pm 1.23$ & $21.30 \pm 2.84$ & $9.83 \pm 3.13$ \\
\hline \multirow[t]{6}{*}{ Female } & $\mathrm{Nw}$ & $9-10$ & $1.31 \pm 0.25$ & $3.42 \pm 0.97$ & $22.63 \pm 3.25$ & $11.70 \pm 2.01$ \\
\hline & $\mathrm{Ow}-\mathrm{Ob}$ & $9-10$ & $1.10 \pm 0.22$ & $3.69 \pm 0.91$ & $23.72 \pm 2.60$ & $13.43 \pm 4.20$ \\
\hline & $\mathrm{Nw}$ & $11-12$ & $1.26 \pm 0.20$ & $3.72 \pm 0.87$ & $22.68 \pm 2.86$ & $11.80 \pm 1.86$ \\
\hline & $\mathrm{Ow}-\mathrm{Ob}$ & $11-12$ & $1.20 \pm 0.17$ & $4.55 \pm 1.12$ & $24.33 \pm 3.12$ & $13.01 \pm 1.59$ \\
\hline & $\mathrm{Nw}$ & $13-14$ & $1.39 \pm 0.23$ & $4.65 \pm 1.04$ & $23.01 \pm 5.70$ & $10.87 \pm 2.53$ \\
\hline & $\mathrm{Ow}-\mathrm{Ob}$ & $13-14$ & $1.24 \pm 0.26$ & $4.78 \pm 1.09$ & $23.40 \pm 2.77$ & $11.80 \pm 3.16$ \\
\hline
\end{tabular}

Table 3. Significant differences between groups according to BMI.

\begin{tabular}{cccccccccccc}
\hline & & & \multicolumn{2}{c}{ SBJ } & \multicolumn{2}{c}{ MBT } & \multicolumn{1}{c}{$10 \times 5$} & \multicolumn{2}{c}{ One Mile RWt } \\
\hline \multirow{2}{*}{ Gender } & Group & Age & $\boldsymbol{d}$ & $\boldsymbol{p}$ & $\boldsymbol{d}$ & $\boldsymbol{p}$ & $\boldsymbol{d}$ & $\boldsymbol{p}$ & $\boldsymbol{d}$ & $\boldsymbol{p}$ \\
\hline \multirow{2}{*}{ Male } & Nw/Ow-Ob & $9-10$ & 1.27 & 0.000 & 0.30 & 0.170 & 0.45 & 0.047 & 0.60 & 0.008 \\
& Nw/Ow-Ob & $11-12$ & 0.60 & 0.005 & 0.48 & 0.024 & 0.50 & 0.019 & 0.93 & 0.000 \\
& Nw/Ow-Ob & $13-14$ & 0.98 & 0.000 & 0.08 & 0.674 & 0.42 & 0.047 & 0.44 & 0.038 \\
\multirow{2}{*}{ Female } & Nw/Ow-Ob & $9-10$ & 0.86 & 0.000 & 0.28 & 0.195 & 0.35 & 0.105 & 0.55 & 0.012 \\
& Nw/Ow-Ob & $11-12$ & 0.60 & 0.207 & 0.83 & 0.000 & 0.54 & 0.014 & 0.66 & 0.003 \\
& Nw/Ow-Ob & $13-14$ & 0.63 & 0.003 & 0.12 & 0.541 & 0.07 & 0.701 & 0.32 & 0.113 \\
\hline
\end{tabular}


Post-hoc analysis in Table 5 evidence significant differences in motor abilities evolution. SBJ and MBT show an increasing score from 9 - 10 yrs to 13 - 14 yrs in $\mathrm{Nw}$ and $\mathrm{Ow}-\mathrm{Ob}$ group, both in male and female (even if girls reported less evidence than boys).

No significant differences were given by $10 \times 5$ and One Mile RWt. Multiple regression analysis, shown in Table 6 , confirmed height and weight as predictors for SBJ and MBT $\left(\mathrm{R}^{2}=0.207, \mathrm{R}^{2}=318\right)$, explaining only $4.0 \%$ and $6.3 \%$ of the variance in $10 \times 5$ and OneMile $\mathrm{RWt}$.

Table 4. Factorial ANOVA between.

\begin{tabular}{ccccccccc}
\hline & \multicolumn{2}{c}{ Gender } & \multicolumn{2}{c}{ BMI } & \multicolumn{2}{c}{ Age } & \multicolumn{2}{c}{ Gender * BMI * Age } \\
\hline & $p$ & $\eta_{p}^{2}$ & $p$ & $\eta_{p}^{2}$ & $p$ & $\eta_{p}^{2}$ & $p$ & $\eta_{p}^{2}$ \\
\hline SBJ & 0.000 & 0.151 & 0.000 & 0.126 & 0.000 & 0.090 & 0.673 & 0.001 \\
MBT & 0.000 & 0.126 & 0.000 & 0.026 & 0.000 & 0.256 & 0.720 & 0.001 \\
$10 \times 5$ & 0.000 & 0.079 & 0.000 & 0.030 & 0.239 & 0.005 & 0.779 & 0.001 \\
One Mile RWt & 0.000 & 0.153 & 0.000 & 0.072 & 0.000 & 0.046 & 0.567 & 0.002 \\
\hline
\end{tabular}

Table 5. Post hoc test showing significant evolution in motor abilities.

\begin{tabular}{|c|c|c|c|c|c|c|}
\hline & \multicolumn{6}{|c|}{ Male } \\
\hline & \multicolumn{3}{|c|}{$\mathrm{Nw}$} & \multicolumn{3}{|c|}{$\mathrm{Ow}-\mathrm{Ob}$} \\
\hline & $10-12$ yrs & $12-14$ yrs & $10-14$ yrs & $10-12$ yrs & $12-14$ yrs & $10-14$ yrs \\
\hline SBJ & 0.684 & 0.000 & 0.000 & 0.016 & 0.075 & 0.000 \\
\hline MBT & 0.021 & 0.000 & 0.000 & 0.000 & 0.001 & 0.000 \\
\hline $10 \times 5$ & 0.704 & 0.592 & 0.144 & 0.837 & 0.417 & 0.209 \\
\hline \multirow[t]{4}{*}{ One Mile RWt } & 0.335 & 0.499 & 0.019 & 0.989 & 0.043 & 0.042 \\
\hline & \multicolumn{6}{|c|}{ Female } \\
\hline & \multicolumn{3}{|c|}{$\mathrm{Nw}$} & \multicolumn{3}{|c|}{ Ow-Ob } \\
\hline & $10-12$ yrs & $12-14$ yrs & $10-14$ yrs & $10-12$ yrs & $12-14$ yrs & $10-14$ yrs \\
\hline SBJ & 0.435 & 0.008 & 0.174 & 0.143 & 0.822 & 0.031 \\
\hline MBT & 0.229 & 0.000 & 0.000 & 0.004 & 0.609 & 0.000 \\
\hline $10 \times 5$ & 0.998 & 0.875 & 0.904 & 0.663 & 0.358 & 0.881 \\
\hline One Mile RWt & 0.970 & 0.064 & 0.107 & 0.849 & 0.252 & 0.083 \\
\hline
\end{tabular}

Table 6. Multipe regression analysis.

\begin{tabular}{cccccccc}
\hline & Weight ${ }^{*}$ Height & \multicolumn{3}{c}{ Weight } & \multicolumn{3}{c}{ Height } \\
\hline $\mathbf{R}^{2}$ & $\mathbf{B}$ & $\mathbf{r}$ & $\mathbf{s r}$ & $\mathbf{B}$ & $\mathbf{r}$ & $\mathbf{s r}$ \\
\hline SBJ & $0.207^{* *}$ & -0.455 & -0.370 & -0.355 & 0.570 & 0.447 & 0.445 \\
MBT & $0.318^{* *}$ & 0.173 & 0.161 & 0.135 & 0.439 & 0.384 & 0.343 \\
10 & $0.040^{* *}$ & 0.250 & 0.195 & 0.194 & -0.205 & -0.161 & -0.160 \\
One Mile RWt & $0.063^{* *}$ & 0.314 & 0.245 & 0.245 & -0.253 & -0.200 & -0.198 \\
\hline
\end{tabular}

${ }^{* *}=p<0.01$. 


\section{Discussion}

The systematic assessment of children's physical activity and physical fitness levels together with the motor learning processes, allows to acquire transversal and longitudinal information on the evolution of motor skills and abilities, necessary to structure and adapt educational process. The quantitative and qualitative data acquired can be used to carry out systematic monitoring of adolescent motor development and "surveillance" studies, necessary to evaluate multi-component interventions, promoting awareness of their own health condition in the students.

Results confirm previous studies highlighting that overweight and obese children and adolescents show lower motor performances than normal weight peers (Grao-Cruces et al., 2018; Kwieciński et al., 2018; Abdelkarim et al., 2019) in all tests except in the medicine ball throw test (MBT). Xu et al. (2020) evidenced better performances in lower limb strength, flexibility, agility and cardiorespiratory fitness, in normal weight adolescents than the obese peers.

This study evidences a significant increase in SBJ and MBT in about all age groups, both in male and female, normal weight and overweight/obese. No statistical differences were carried out regarding the evolution of $10 \times 5$ and OneMile RWt in different ages. Moreover, data evidence that weight and height are important predictors for SBJ and MBT test, explaining the $20.7 \%$ and $31.8 \%$ of the total variance, respectively. This suggests that strength test in which are required single movement execution are more associated and conditioned by individual growth and anthropometrics factors, than test required repeated movement and greater coordinative engagement (such as $10 \times 5$ and One Mile Test).

Kalac and Gontarev (2015) evidenced that voluminosity, associated with a high percentage of fat tissue, is negatively associated with coordination, agility and speed, and muscle mass is positively correlated with the explosive strength of the lower extremities. Ito et al. (2020) found that weight-height ratio was correlated with acute aerobic exercise and endurance exercise (more markedly for boys). Fiori et al. (2020) affirmed that 1 ) the effect of body mass index on children's physical fitness intensifies with age, and 2) overweight and obesity are inversely correlated with aerobic capacity, agility, lower limb strength and balance but positively correlated with upper limb strength.

Several studies evidenced that MVPA (moderate to vigorous physical activity) is strongly associated with motor performances, and inversely related to adiposity (Júdice et al., 2017; Santos et al., 2019; Jones et al., 2020). Interventions aimed at improvement of resistance and cardiorespiratory fitness in youth and adolescents are often based on the amount of daily/weekly physical activity (3 - 5 times a week), and the evolution in motor performances is dose-effect dependent (Brustio et al., 2020; Martin-Smith et al., 2020; Nevill et al., 2020).

Another important element to consider to interpretate the no significant differences in motor abilities evolution is the quality of movement. Molina-Garcia et al. (2019) suggest that children with high BMI demonstrate lower functional 
movement quality independently of fitness level, whereas children with better fitness level (i.e., cardiorespiratory fitness, lower limbs muscular strength, and speed-agility) demonstrate greater functional movement quality independently of fatness level. Findings showed better motor coordination improvements and performances in children and adolescents with slower increasing BMI, suggesting a decrease in motor competences with an increase in BMI (Lopes et al., 2020; Lopes et al., 2018).

\section{Conclusion}

The study can provide some methodological indications necessary to raise the quality of teaching in PE. The data show a nonlinear and significant evolution of motor performances and abilities from $9-10$ yrs to 13 - 14 yrs, despite differences between normal weight and overweight/obese group.

Reduced motor performances should be considered the result, and not the cause, of a process strictly related with two other factors:

- Decrease in MVPA in children and adolescents, both normal weight and overweight children;

- Reduction of movement quality and proficiency, according to less levels of motor coordination.

Results show that physical fitness is strictly correlated to anthropometric factors during single strength test, while speed and endurance tests are less correlated to anthropometric factors. This implies that, in order to adequately develop the levels of physical fitness, it is not necessary to make students exercise repeatedly and in a given task only, for example run for 20 minutes, perform a given circuit three times, or perform 10 push up, etc. Certainly, this method of teaching-learning, based on the mechanical repetition of tasks, favors the development, even quite rapid, of motor skills, but what happens to the qualitative learning process of movement? And physical activity levels? Can every one perform and complete the tasks proposed by the teacher?

The following implications for practice can be inferred. Teacher should enable children to perform all the proposed motor tasks, adapting the activities to the motor skills of children with a high BMI, in the parameters of executive difficulty, duration and intensity.

In this way, teacher increases both quantitative levels of physical activity, and quality of motor skills learning, promoting success and motivation, fundamental factors to ensure the adherence to physical activity into adult age. The use of different teaching styles could help teacher to modulate and adapt some variants of movement (space, setting, duration, playing field, rules, tools, etc.), to allow children to participate in activities successfully, and increase time spent in physical activity (Myer et al., 2015).

The introduction of new and different opportunities to be physically active during, before and after school day, for examples in the form of active breaks, active lessons, increasing the number of $\mathrm{PE}$ in the curricula, active transport, 
etc., could be viable solutions to increase physical activity levels, both in non-structured (play) and structured form (game).

Teachers should encourage this type of non-linear approach and learning, as it represents the basis and fundamental prerequisite for any other type of motor learning, physical fitness development and for reaching the individual and personal stages of proximal development in physical education.

\section{Conflicts of Interest}

The authors declare no conflicts of interest regarding the publication of this paper.

\section{References}

Abarca-Gómez, L., Abdeen, Z. A., Hamid, Z. A., Abu-Rmeileh, N. M., Acosta-Cazares, B., Acuin, C. et al. (2017). Worldwide Trends in Body-Mass Index, Underweight, Overweight, and Obesity from 1975 to 2016: A Pooled Analysis of 2416 Population-Based Measurement Studies in 128.9 Million Children, Adolescents, and Adults. The Lancet, 390, 2627-2642. https://doi.org/10.1016/S0140-6736(17)32129-3

Abdelkarim, O., Ammar, A., Trabelsi, K., Cthourou, H., Jekauc, D., Irandoust, K. et al. (2019). Prevalence of Underweight and Overweight and Its Association with Physical Fitness in Egyptian Schoolchildren. International Journal of Environmental Research and Public Health, 17, Article No. 75. https://doi.org/10.3390/ijerph17010075

Abrignani, M. G., Lucà, F., Favilli, S., Benvenuto, M., Rao, C. M., Di Fusco, S. A. et al. (2019). Lifestyles and Cardiovascular Prevention in Childhood and Adolescence. Pediatric Cardiology, 40, 1113-1125. https://doi.org/10.1007/s00246-019-02152-w

Al-Khudairy, L., Loveman, E., Colquitt, J. L., Mead, E., Johnson, R. E., Fraser, H. et al. (2017). Diet, Physical Activity and Behavioural Interventions for the Treatment of Overweight or Obese Adolescents Aged 12 to 17 years. The Cochrane Database of Systematic Reviews, 6, Article ID: CD012691. https://doi.org/10.1002/14651858.CD012691

Alves, J. G. B., \& Alves, G. V. (2019). Effects of Physical Activity on Children's Growth. Jornal de Pediatria, 95, 72-78. https://doi.org/10.1016/j.jped.2018.11.003

Bhaskaran, K., Dos-Santos-Silva, I., Leon, D. A., Douglas, I. J., \& Smeeth, L. (2018). Association of BMI with Overall and Cause-Specific Mortality: A Population-Based Cohort Study of 3.6 Million Adults in the UK. The Lancet. Diabetes \& Endocrinology, 6, 944-953. https://doi.org/10.1016/S2213-8587(18)30288-2

Brustio, P. R., Mulasso, A., Lupo, C., Massasso, A., Rainoldi, A., \& Boccia, G. (2020). The Daily Mile Is Able to Improve Cardiorespiratory Fitness When Practiced Three Times a Week. International Journal of Environmental Research and Public Health, 17, Article No. 2095. https://doi.org/10.3390/ijerph17062095

Bull, F. C., Al-Ansari, S. S., Biddle, S., Borodulin, K., Buman, M. P., Cardon, G. et al. (2020). World Health Organization 2020 Guidelines on Physical Activity and Sedentary Behaviour. British Journal of Sports Medicine, 54, 1451-1462. https://doi.org/10.1136/bjsports-2020-102955

Cohen, J. (1988). Statistical Power Analysis for the Behavioral Sciences (2nd ed.). Hillsdale, NJ: Lawrence Erlbaum Associates

Cole, T. J., Bellizzi, M. C., Flegal, K. M., \& Dietz, W. H. (2000). Establishing a Standard Definition for Child Overweight and Obesity Worldwide: International Survey. BMJ, 320, 1240-1243. https://doi.org/10.1136/bmj.320.7244.1240 
Colley, R. C., Clarke, J., Doyon, C. Y., Janssen, I., Lang, J. J., Timmons, B. W., \& Tremblay, M. S. (2019). Trends in Physical Fitness among Canadian Children and Youth. Health Reports, 30, 3-13. https://doi.org/10.25318/82-003-x201901000001-eng

Dong, Y., Lau, P. W. C., Dong, B., Zou, Z., Yang, Y., Wen, B. et al. (2019). Trends in Physical Fitness, Growth, and Nutritional Status of Chinese Children and Adolescents: A Retrospective Analysis of 1.5 Million Students from Six Successive National Surveys between 1985 and 2014. The Lancet: Child \& Adolescent Health, 3, 871-880. https://doi.org/10.1016/S2352-4642(19)30302-5

Faigenbaum, A. D., Rebullido, T. R., \& MacDonald, J. P. (2018). Pediatric Inactivity Triad: A Risky PIT. Current Sports Medicine Reports, 17, 45-47. https://doi.org/10.1249/JSR.0000000000000450

Fiori, F., Bravo, G., Parpinel, M., Messina, G., Malavolta, R., \& Lazzer, S. (2020). Relationship between Body Mass Index and Physical Fitness in Italian Prepubertal Schoolchildren. PLoS ONE, 15, e0233362. https://doi.org/10.1371/journal.pone.0233362

Grao-Cruces, A., Ruiz-Ariza, A., De La Torre-Cruz, M. J., \& Martínez-López, E. J. (2018). Students with Excess Weight Obtain Lower Physical Fitness Test Scores in Physical Education than Normal Weight Pairs: Myth or Reality? Journal of Physical Education \& Sport, 18, 1562-1571.

Guthold, R., Stevens, G. A., Riley, L. M., \& Bull, F. C. (2020). Global Trends in Insufficient Physical Activity among Adolescents: A Pooled Analysis of 298 Population-Based Surveys with 1.6 Million Participants. The Lancet: Child \& Adolescent Health, 4, 23-35. https://doi.org/10.1016/S2352-4642(19)30323-2

Hosker, D. K., Elkins, R. M., \& Potter, M. P. (2019). Promoting Mental Health and Wellness in Youth through Physical Activity, Nutrition, and Sleep. Child and Adolescent Psychiatric Clinics of North America, 28, 171-193. https://doi.org/10.1016/j.chc.2018.11.010

Ito, S., Fukumoto, T., \& Imagita, H. (2020). Relation between the Height-Weight ratio and physical fitness among Japanese elementary school students. Journal of Physical Education \& Sport, 20, 690-706. https://doi.org/10.1080/02640414.2019.1685842

Jones, M. A., Skidmore, P. M., Stoner, L., Harrex, H., Saeedi, P., Black, K., \& Barone Gibbs, B. (2020). Associations of Accelerometer-Measured Sedentary Time, Sedentary Bouts, and Physical Activity with Adiposity and Fitness in Children. Journal of Sports Sciences, 38, 114-120. https://doi.org/10.1080/02640414.2019.1685842

Júdice, P. B., Silva, A. M., Berria, J., Petroski, E. L., Ekelund, U., \& Sardinha, L. B. (2017). Sedentary Patterns, Physical Activity and Health-Related Physical Fitness in Youth: A Cross-Sectional Study. The International Journal of Behavioral Nutrition and Physical Activity, 14, Article No. 25. https://doi.org/10.1186/s12966-017-0481-3

Kalac, R., \& Gontarev, S. (2015). Cross-Correlation between the Anthropometric Measures and Motor Tests for Student from 6 to 14 Years. Research in Physical Education, Sport \& Health, 4, 85-93.

Kwieciński, J., Konarski, J. M., Strzelczyk, R., Krzykała, M., Konarska, A., Bartkowiak, S. et al. (2018). Non-Linear Relationships between the BMI and Physical Fitness in Polish Adolescents. Annals of Human Biology, 45, 406-413. https://doi.org/10.1080/03014460.2018.1494306

Lopes, V. P., Malina, R. M., Maia, J. A. R., \& Rodrigues, L. P. (2018). Body Mass Index and Motor Coordination: Non-Linear Relationships in Children 6 - 10 Years. Child: Care, Health and Development, 44, 443-451. https://doi.org/10.1111/cch.12557

Lopes, V. P., Utesch, T., \& Rodrigues, L. P. (2020). Classes of Developmental Trajectories of Body Mass Index: Differences in Motor Competence and Cardiorespiratory Fitness. 
Journal of Sports Sciences, 38, 619-625. https://doi.org/10.1080/02640414.2020.1722024

Martin-Smith, R., Cox, A., Buchan, D. S., Baker, J. S., Grace, F., \& Sculthorpe, N. (2020). High Intensity Interval Training (HIIT) Improves Cardiorespiratory Fitness (CRF) in Healthy, Overweight and Obese Adolescents: A Systematic Review and Meta-Analysis of Controlled Studies. International Journal of Environmental Research and Public Health, 17, Article No. 2955. https://doi.org/10.3390/ijerph17082955

Milanovic, I., Radisavljevic-Janic, S., Zivkovic, M. Z., \& Mirkov, D. M. (2019). Health-Related Physical Fitness Levels and Prevalence of Obesity in Serbian Elementary Schoolchildren. Nutricion Hospitalaria, 36, 245-296.

Molina-Garcia, P., H Migueles, J., Cadenas-Sanchez, C., Esteban-Cornejo, I., Mora-Gonzalez, J., Rodriguez-Ayllon, M. et al. (2019). Fatness and Fitness in Relation to Functional Movement Quality in Overweight and Obese Children. Journal of Sports Sciences, 37, 878-885. https://doi.org/10.1080/02640414.2018.1532152

Myer, G. D., Faigenbaum, A. D., Edwards, N. M., Clark, J. F., Best, T. M., \& Sallis, R. E. (2015). Sixty Minutes of What? A Developing Brain Perspective for Activating Children with an Integrative Exercise Approach. British Journal of Sports Medicine, 49, 1510-1516. https://doi.org/10.1136/bjsports-2014-093661

Nevill, A. M., Duncan, M. J., \& Sandercock, G. (2020). The Dose-Response Association between $\mathrm{VO}_{2 \text { peak }}$ and Self-Reported Physical Activity in Children. Journal of Sports Sciences, 38, 1829-1835. https://doi.org/10.1080/02640414.2020.1756682

Pate, R. R., Schenkelberg, M. A., Dowda, M., \& McIver, K. L. (2019). Group-Based Physical Activity Trajectories in Children Transitioning from Elementary to High School. BMC Public Health, 19, Article No. 323. https://doi.org/10.1186/s12889-019-6630-7

Pierce, C. A., Block, R. A., \& Aguinis, H. (2004). Cautionary Note on Reporting Eta-Squared Values From Multifactor ANOVA Designs. Educational and Psychological Measurement, 64, 916-924. https://doi.org/10.1177/0013164404264848

Rodrigues, L. P., Stodden, D. F., \& Lopes, V. P. (2016). Developmental Pathways of Change in Fitness and Motor Competence Are Related to Overweight and Obesity Status at the End of Primary School. Journal of Science and Medicine in Sport, 19, 87-92. https://doi.org/10.1016/j.jsams.2015.01.002

Santos, D. A., Magalhães, J. P., Júdice, P. B., Correia, I. R., Minderico, C. S., Ekelund, U., \& Sardinha, L. B. (2019). Fitness Mediates Activity and Sedentary Patterns Associations with Adiposity in Youth. Medicine and Science in Sports and Exercise, 51, 323-329. https://doi.org/10.1249/MSS.0000000000001785

Singh, A. S., Saliasi, E., van den Berg, V., Uijtdewilligen, L., de Groot, R. H. M., Jolles, J. et al. (2019). Effects of Physical Activity Interventions on Cognitive and Academic Performance in Children and Adolescents: A Novel Combination of a Systematic Review and Recommendations from an Expert Panel. British Journal of Sports Medicine, 53, 640-647. https://doi.org/10.1136/bjsports-2017-098136

Utesch, T., Bardid, F., Büsch, D., \& Strauss, B. (2019). The Relationship between Motor Competence and Physical Fitness from Early Childhood to Early Adulthood: A Meta-Analysis. Sports Medicine, 49, 541-551. https://doi.org/10.1007/s40279-019-01068-y

Xu, Y., Mei, M., Wang, H., Yan, Q., \& He, G. (2020). Association between Weight Status and Physical Fitness in Chinese Mainland Children and Adolescents: A Cross-Sectional Study. International Journal of Environmental Research and Public Health, 17, Article No. 2468. https://doi.org/10.3390/ijerph17072468

Yu, J., Tao, Y., Dou, J., Ye, J., Yu, Y., \& Jin, L. (2018). The Dose-Response Analysis between BMI and Common Chronic Diseases in Northeast China. Scientific Reports, 8 , Article No. 4228. https://doi.org/10.1038/s41598-018-22551-y 
Yuksel, H. S., Şahin, F. N., Maksimovic, N., Drid, P., \& Bianco, A. (2020). School-Based Intervention Programs for Preventing Obesity and Promoting Physical Activity and Fitness: A Systematic Review. International Journal of Environmental Research and Public Health, 17, Article No. 347. https://doi.org/10.3390/ijerph17010347

Zhang, T., Xu, J., Li, S., Bazzano, L. A., He, J., Whelton, P. K., \& Chen, W. (2019). Trajectories of Childhood BMI and Adult Diabetes: the Bogalusa Heart Study. Diabetologia, 62, 70-77. https://doi.org/10.1007/s00125-018-4753-5 